\title{
Experimental and Numerical Investigation of Cold Bulge Forming of Titanium Alloy Ti55
}

\author{
S. M. R. Tabatabaei ${ }^{*}$, M. Safari ${ }^{2}$, R. Shahrokh Esfahani ${ }^{2}$, \\ A. Abolghasemian. Sichani ${ }^{3}$, M. Nourjani Pour Moghadam ${ }^{4}$ \\ ${ }^{1}$ Department of Mechanical Engineering, Razi University of Kermanshah, Kermanshah, Iran \\ ${ }^{2}$ Department of Mechanical Engineering, Isfahan University of Technology, Isfahan, Iran \\ ${ }^{3}$ Department of Mechanical Engineering, Khomeinishahr Branch, Islamic Azad University, Isfahan, Iran \\ ${ }^{4}$ Department of Mechanical Engineering, Amir Kabir University of Technology, Tehran, Iran \\ Email: *mitso64sed@yahoo.com
}

Received July 31, 2013; revised September 2, 2013; accepted October 1, 2013

Copyright (c) 2013 S. M. R. Tabatabaei et al. This is an open access article distributed under the Creative Commons Attribution License, which permits unrestricted use, distribution, and reproduction in any medium, provided the original work is properly cited. In accordance of the Creative Commons Attribution License all Copyrights (C) 2013 are reserved for SCIRP and the owner of the intellectual property S. M. R. Tabatabaei et al. All Copyright (C) 2013 are guarded by low and by SCIRP as a guardian.

\begin{abstract}
In this paper, cold bulge forming of titanium alloy Ti55 was investigated. This process was done successfully and titanium alloy Ti55 was formed completely. Also, in the numerical work, this process has been investigated using Abaqus/ Explicit code. The results show that there is a good agreement between experimental and numerical results. Mechanical properties of Titanium alloy Ti55 have been investigated before and after bulge forming. The results show that mechanical properties of titanium alloy Ti55 have been improved during bulge forming. Also, hardness, and thickness variation tests of sheet metal before and after bulge forming were performed and the same results to mechanical properties were obtained.
\end{abstract}

Keywords: Cold Bulge Forming; Titanium Alloy Ti55; Abaqus/Explicit

\section{Introduction}

These days Sheet metal parts are made of titanium and its alloys are used extensively in the aircraft industry due to their good strength-to-weight ratio compared to steel and aluminum, corrosion resistance (because of the thin adherent oxide coating), and high mechanical strength at elevated temperatures. Many titanium sheet metal parts used in aircraft consist of straight bends (for example, ribs, and stringers). So, most titanium alloys need to be hot worked (that is, plastically deformed above the material's recrystallization temperature) since their tendency to strain harden at lower temperatures and their alphabeta microstructure is extremely sensitive to temperature (e.g., Ti-6Al-4V).

Bulge forming is a widely used industrial process which is generally utilized to form complex components from tubular blanks. Components are formed by restraining the blank in a die bearing the desired shape and applying an internal hydrostatic pressure to the tube via a

\footnotetext{
${ }^{*}$ Corresponding author.
}

liquid or solid medium. Bulge forming by pressure load alone is limited in the type and quality of components that can produce. If a compressive axial load is applied to the ends of the tube in conjunction with the pressure load, the material can be pushed into the deformation zone during forming, thus preventing excessive thinning of the walls.

In 2001, Mac Donald et al. [1] presented a three-dimensional simulation of the manufacture of cross-branch components using a solid bulging medium. They also investigated the effect of varying friction between the bulging medium and the tube and presented the history of development of the bulge and stress conditions in the formed component. In 2002, Mac Donald et al. [2] presented finite element models to simulate axisymmetric tube bulging and hydraulic bulge forming of cross branches from a straight tube, with a particular emphasis on the state of stress and deformation of the die. They investigated effects of using internal pressure alone and combined internal pressure and axial compressive load and found that the development of stress and the location of 
maximum stress was dependant on the type of loading used. In 2004, Hou et al. [3] manufactured by bulge forming 1 and $4 \mathrm{~mm}$ thick hemispherical parts and basinshaped parts of PC based on experimental data concerning the tensile mechanical properties of polycarbonate at high temperature. In their work, Numerical simulation for PC bulge-forming has been performed with the software DYNAFORM and a material model for PC has been established. The experimental data agree well with that of numerical simulation. Their results showed that the thinnest part of the hemisphere is at the bottom, the side walls gradually thickening to a flat curve. The thinnest region of the basin-shaped part is the point linking the bottom and the side walls. Consequently, the thickness at the bottom is uniform while the thickness of side wall changes. In 2010, Zheng et al. [4] investigated the micro-scale laser bulge forming process using both numerical and experimental methods. In their work, the effect of laser energy on microformability of pure copper was discussed in detail. Also a 3D measuring laser microscope was adopted to measure deformed regions under different laser energy levels. The deformation measurements showed that the experimental and numerical results were in good agreement. In 1998, Lee et al. [5] have made an analytical model reflecting the microstructural evolution on the basis of Dutta and Mukherjee's work, and used this model to the superplastic bulge forming of Ti-6Al-4V alloy. In their work, bulge forming of fine grain $(2.5 \mathrm{~mm}) \mathrm{Ti}-6 \mathrm{Al}-4 \mathrm{~V}$ alloy sheets was conducted at $900^{\circ} \mathrm{C}$, at three strain rates. It was found that the grain growth rate of biaxially bulge formed samples was quite different from that of uniaxially deformed specimens and that the grain growth rate was independent of the strain rate imposed at a fixed temperature. After incorporating the grain growth rate of biaxially deformed material into the analytical model, the bulge forming process was successfully controlled at different strain rates. Prediction of the thickness distribution by the modified model was in agreement with the experimental results. In 2000, Mac Donald et al. [6] presented a finite element simulation of the manufacture of cross branches from straight tubes, using the bulge forming method, and investigated the effects of varying process parameters. In their work, two different types of loading were employed: internal pressure load only and combined internal pressure and axial loading. The effects of friction and tube thickness variation are examined, and deformations, stresses and tube thinning/thickening behavior in the formed component are presented. In 2002, Mac Donald et al. [7] in a review discussed the application of bulge forming to the manufacture of near-net-shape components and discussed machine and tool design.

In the present work, an attempt is made to investigate the cold bulge forming of titanium alloy Ti55. This pro- cess was done successfully and titanium alloy Ti55 was formed completely. Also, in a numerical work, this process has been investigated using Abaqus/Explicit code. The results show that there is a good agreement between experimental and numerical results. Moreover, Mechanical properties of Titanium alloy Ti55 have been investigated before and after bulge forming. The results show that mechanical properties of titanium alloy Ti55 have been improved during bulge forming. Also, hardness and thickness variation tests of sheet metal before and after bulge forming were performed and the same results to mechanical properties were obtained.

\section{Experimental Work}

The material used in this study was Titanium alloy Ti55 with thickness $0.5 \mathrm{~mm}$. Preliminary blanks were trimmed according to a pattern and then rolled in a CNC roller. In Figure 1, steps of preparing the blanks are shown. After preparing the blank, bulge forming of titanium alloy $\mathrm{Ti}$ 55 was performed using a urethane rubber as a solid medium. For this purpose, a 25 tone hydraulic press was used. In Figure 2 schematic of forming tools used in this work is shown. Also, in Figure 3, deformed titanium blank in bulge forming process is shown. As it is shown in Figure 3, titanium blank Ti55 has been formed successfully in the cold bulge forming process. Therefore, it is demonstrated that titanium blank Ti55 can be formed using bulge forming process without pre-heating the titanium blank. Hence, this success can be used in the industrial applications for titanium alloys.

\section{Numerical Work}

In the numerical work, simulations have been done using commercially available finite element code ABAQUS/ Explicit. In the simulations, the punch and die were modeled as rigid bodies while the urethane and titanium blank were modeled as deformable volume and deformable shell respectively. It is done a new work in the simulation of urethane in this paper. Additionally, a new method is invented in this contribution for simulation of urethane. Mechanical properties of urethane in the Abaqus/Explicit were employed as a hyper elastic material. For this purpose, using Ogden equation and uniaxial test data were used and with software evaluation, mechanical properties of urethane were obtained. The Coulomb friction model with a constant coefficient of friction, $\mu=0.3$ was applied. In Figure 4 graphical results of stress and strain distribution in the final step of bulge forming of titanium blank is depicted.

\section{Results and Discussion}

After bulge forming of titanium blank, test pieces of deformed blank were trimmed and investigated under ten- 


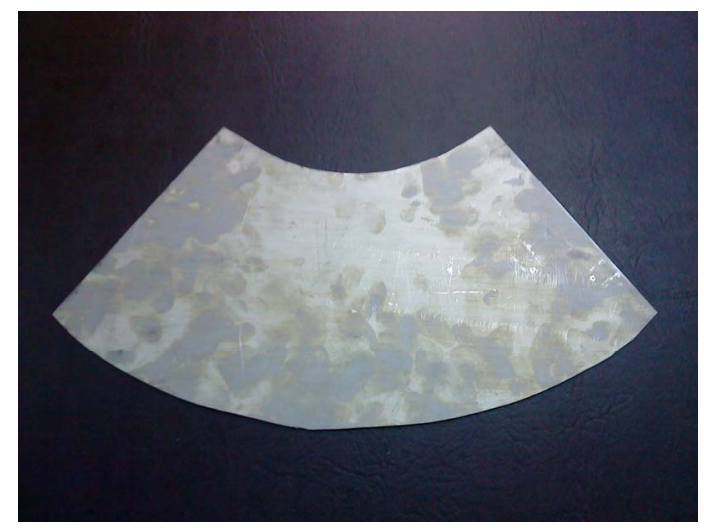

(a)

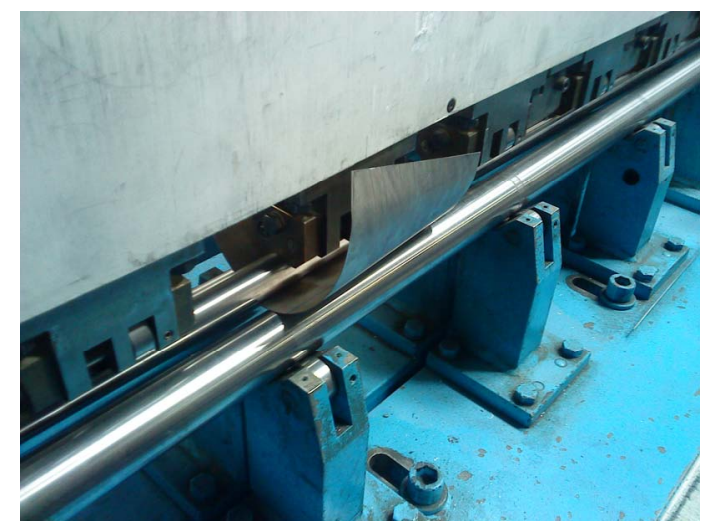

(b)

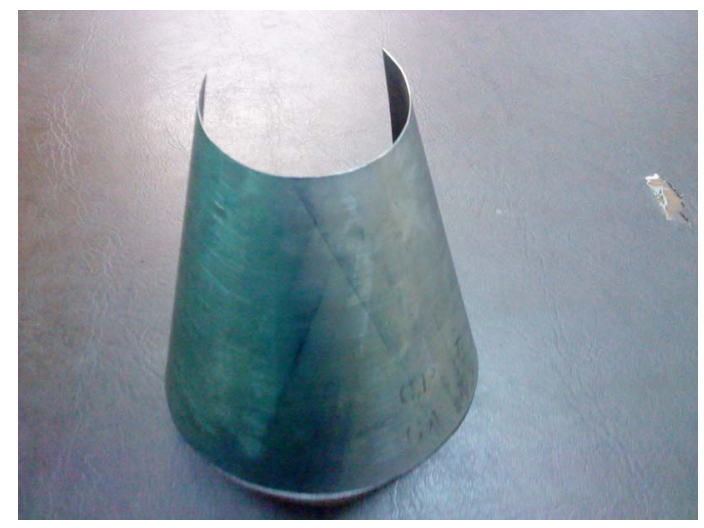

(c)

Figure 1. Steps of preparing of blanks, a-Trimming preliminary blank according to a pattern, b-Rolling the blank in a CNC roller, c-Final blank that is used in the bulge forming.

sion test, hardness test and thickness distribution test. Then the results of these tests were compared with results of same tests obtained from test pieces before bulge forming.

\subsection{Tension Test}

For tension test, a universal press was used. The results of this test are presented in Table 1.

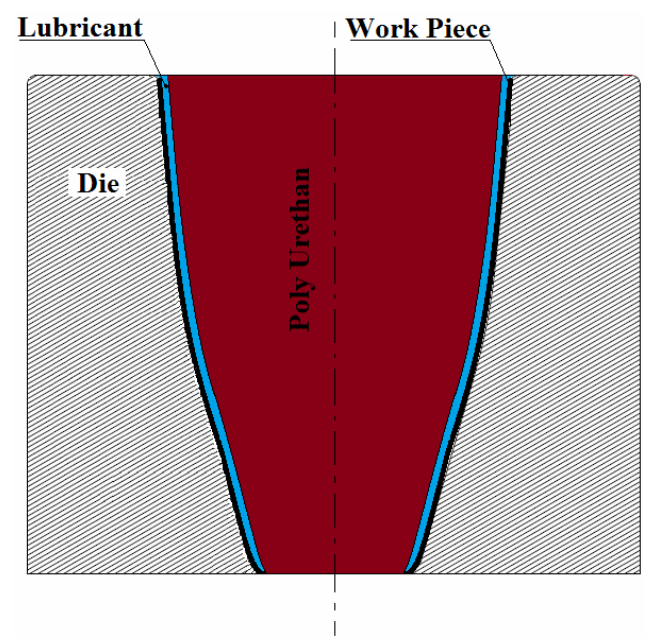

Figure 2. Schematic of forming tools used in this work.

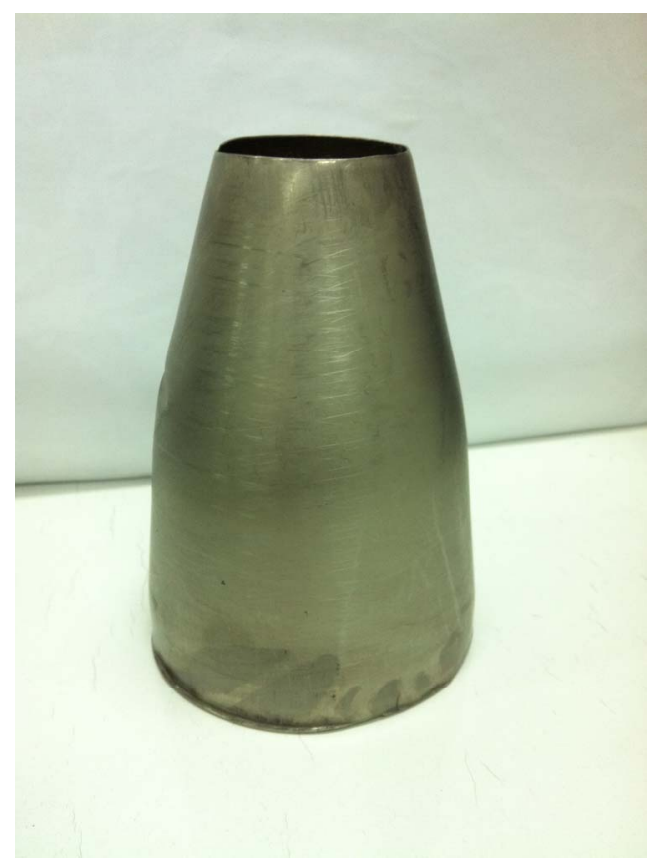

Figure 3. Deformed titanium blank in bulge forming process.

Table 1. Results of tension test.

\begin{tabular}{cccc}
\hline Test piece & Yield stress (MPa) & $\begin{array}{c}\text { Ultimate } \\
\text { stress (MPa) }\end{array}$ & $\begin{array}{c}\text { Elongation } \\
(\%)\end{array}$ \\
\hline Before bulge forming & 400 & 530 & 21 \\
After bulge forming & 500 & 560 & 15.5 \\
\hline
\end{tabular}

As it can be understood from Table 1, strength of titanium blank was increased after bulge forming. Therefore, it is demonstrated that titanium blank has been formed successfully by cold bulge forming. Also, as it is shown in Figure 4 for stress distribution, numerical results are in good agreement with experimental results. As it is 

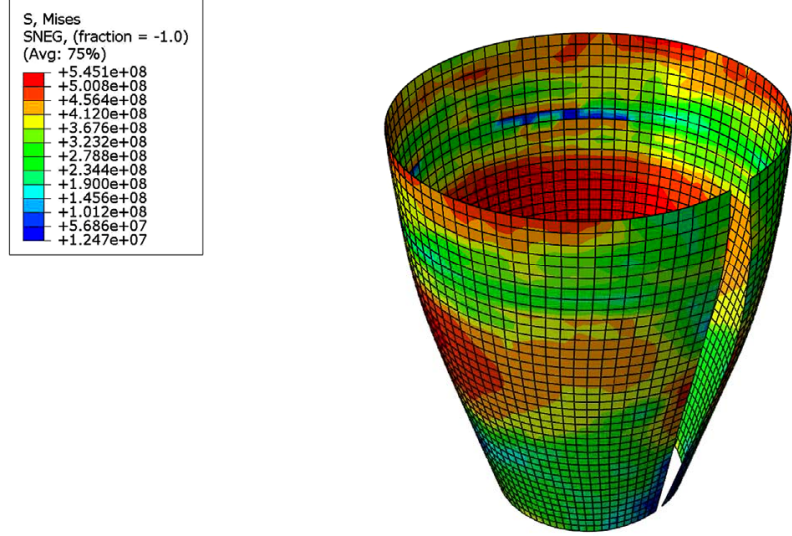

(a)

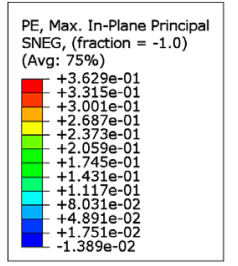

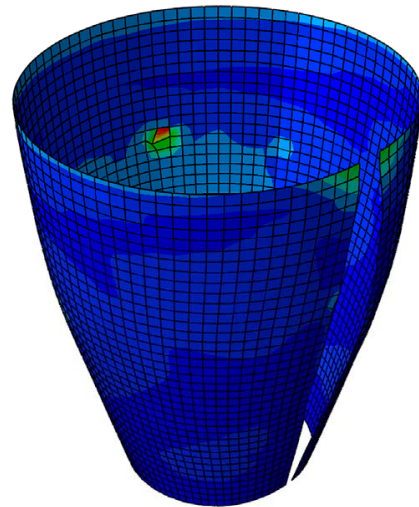

(b)

Figure 4. Graphical results in the final step of bulge forming of titanium blank, a) stress distribution; b) plastic strain distribution.

seen from Table 1, yield and ultimate stresses are increased after bulge forming due to strain hardening. In this state, elongation of work-piece is decreased due to similar reason.

\subsection{Hardness Test}

A device with model Swiss Max 300 hardness tester was applied for hardness test. Obtained harness results for test pieces before and after bulge forming were $180 \mathrm{HV}$ (Vickers hardness) and $187 \mathrm{HV}$ (Vickers hardness) respectively. Therefore, it can be concluded that titanium blank has been formed successfully by cold bulge forming.

\subsection{Thickness Distribution Test}

For this test, a test piece was trimmed in longitudinal direction and thickness distribution along it was measured using an ultrasonic thickness distribution device with model DM4. In the numerical simulation, thickness distribution along the same position and direction was obtained. In Figure 5, experimental and numerical thick- ness distribution is compared.

Based on Figure 5, experimental and numerical results are in good agreement and it is concluded that thickness of titanium blank after bulge forming approximately remained constant.

\section{Conclusion}

In this paper, cold bulge forming of titanium alloy Ti55 has been investigated. This process was done successfully and titanium alloy Ti55 was formed completely. Also, in a numerical work, this process has been investigated using Abaqus/Explicit code. The results show that there is a good agreement between experimental and numerical results. Mechanical properties of Titanium alloy Ti55 have been investigated before and after bulge forming. The results show that mechanical properties of titanium alloy Ti55 have been improved during bulge

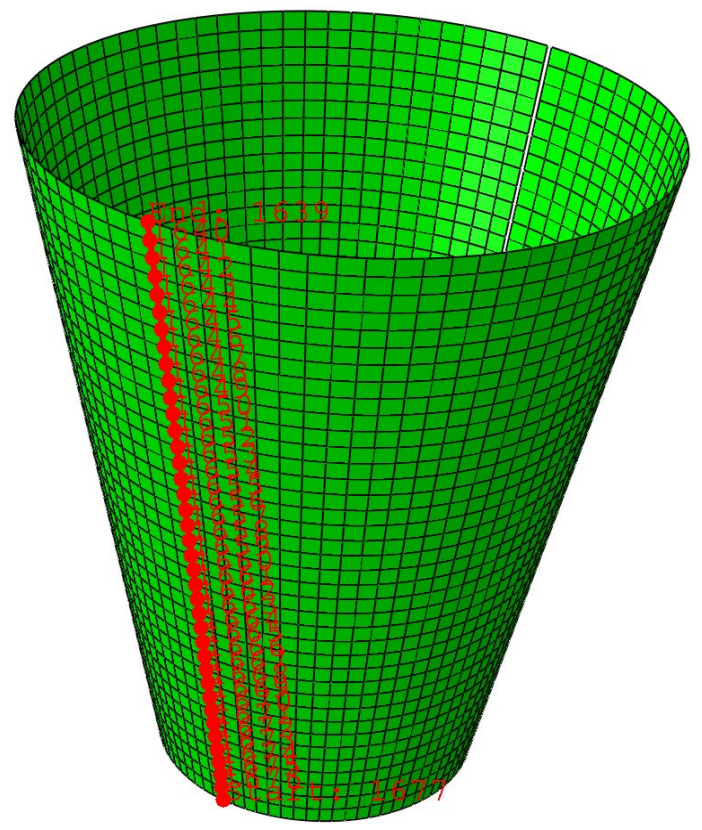

(a)

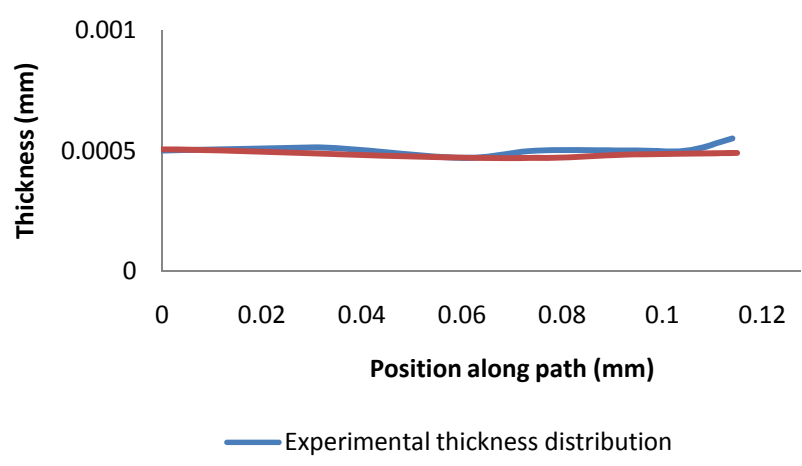

(b)

Figure 5. Thickness distribution, a) path; b) comparison between experimental and numerical results. 
forming. Also, hardness and thickness variation tests of sheet metal before and after bulge forming were performed and the same results to mechanical properties were obtained.

\section{REFERENCES}

[1] B. J. Mac Donald and M. S. J. Hashmi, "Three-Dimensional Finite Element Simulation of Bulge Forming Using a Solid Bulging Medium," Finite Elements in Analysis and Design, Vol. 37, No. 2, 2001, pp. 107-116. http://dx.doi.org/10.1016/S0168-874X(00)00023-8

[2] B. J. Mac Donald and M. S. J. Hashmi, "Analysis of Die Behaviour during Bulge Forming Operations Using the Finite Element Method,” Finite Elements in Analysis and Design, Vol. 39, No. 2, 2002, pp. 137-151. http://dx.doi.org/10.1016/S0168-874X(02)00074-4

[3] Z. X. Hou, J. Wu and Z. R. Wang, "A Study of the BulgeForming of Polycarbonate (PC) Sheet," Journal of Materials Processing Technology, Vol. 151, No. 1-3, 2004, pp. 312-315.

http://dx.doi.org/10.1016/j.jmatprotec.2004.04.080

[4] C. Zheng, S. Sun, Z. Ji and W. Wang, "Effect of Laser
Energy on the Deformation Behavior in Microscale Laser Bulge Forming,” Applied Surface Science, Vol. 257, No. 5, 2010, pp. 1589-1595. http://dx.doi.org/10.1016/j.apsusc.2010.08.099

[5] J. H. Lee, Y. J. Song, D. H. Shin and C. S. Lee, "Microstructural Evolution during Superplastic Bulge Forming of Ti-6Al-4V Alloy,” Materials Science and Engineering, Vol. A243, No. 1-2, 1998, pp. 119-125. http://dx.doi.org/10.1016/S0921-5093(97)00788-0

[6] B. J. Mac Donald and M. S. J. Hashmi, "Finite Element Simulation of Bulge Forming of a Cross-Joint from a Tubular Blank," Journal of Materials Processing Technology, Vol. 103, No. 3, 2000, pp. 333-342. http://dx.doi.org/10.1016/S0924-0136(00)00522-7

[7] B. J. Mac Donald and M. S. J. Hashmi, "Near-Net-Shape Manufacture of Engineering Components Using Bulge Forming Processes: A Review," Journal of Materials Processing Technology, Vol. 120, No. 1-3, 2002, pp. 341-347. http://dx.doi.org/10.1016/S0924-0136(01)01120-7 